\title{
Laser Acceleration of Electron Beams to the GeV-class Energies in Gas Jets
}

\author{
Nasr A. M. Hafz ${ }^{1 *}$, Tae Moon Jeong', Seong Ku Lee ${ }^{1}$, IL Woo Choi ${ }^{1}$, Ki Hong Pae ${ }^{1}$, Victor V. \\ Kulagin $^{2}$, Jae Hee Sung ${ }^{1}$, Tae Jun $\mathrm{Yu}^{1}$, John R. Cary ${ }^{3,4}$, Do-Kyeong Ko ${ }^{5}$, and Jongmin Lee ${ }^{1 \dagger}$ \\ ${ }^{1}$ Center for Femto-Atto Science and Technology (c-FAST), Advanced Photonics Research \\ Institute Gwangju Institute of Science and Technology, Gwangju 500-712, Korea \\ ${ }^{2}$ Sternberg Astronomical Institute, Moscow State University, 119899 Moscow, Russia \\ ${ }^{3}$ Department of Physics, University of Colorado, Boulder, Colorado 80309, USA \\ ${ }^{4}$ Tech-X Corporation, Boulder, Colorado 80303, USA \\ ${ }^{5}$ School of Photon Science and Technology and Advanced Photonics Research Institute \\ Gwangju Institute of Science and Technology, Gwangju 500-712, Korea
}

(Received December 16, 2008 : revised January 8, 2009 : accepted January 8, 2009)

\begin{abstract}
In a laser-plasma wakefield accelerator, the ponderomotive force of an ultrashort high intensity laser pulse excites a longitudinal wave or plasma bubble in a way similar to the excitation of a wake wave behind a boat as it propagates on the water surface. Electric fields inside the plasma bubble can be several orders of magnitude higher than those available in conventional RF-based particle accelerator facilities which are limited by material breakdown. Therefore, if an electron bunch is properly phase-locked with the bubble's acceleration field, it can gain relativistic energies within an extremely short distance. Here, in the bubble regime we show the generation of stable and reproducible sub $\mathrm{GeV}$, and GeV-class electron beams. Supported by three-dimensional particle-in-cell simulations, our experimental results show the highest acceleration gradients produced so far. Simulations suggested that the plasma bubble elongation should be minimized in order to achieve higher electron beam energies.
\end{abstract}

Keywords: Laser acceleration, GeV electron beams, Bubble regime, Particle-in-cell simulations

OCIS codes : (000.2190) Experimental physics; (320.7080) Ultrafast devices; (350.5400) Plasmas, (260.5950) Self-focusing

\section{INTRODUCTION}

Laser-plasma interactions at the relativistic intensity of $\geq 10^{18} \mathrm{~W} / \mathrm{cm}^{2}$ reveal interesting nonlinear optical plasma phenomena $[1,2]$. When an ultrashort terawatt laser pulse is focused in an underdense plasma, the radiation pressure from the laser pulse excites large-amplitude electrostatic plasma waves, which can trap and accelerate electrons to relativistic energies in short distances. This is known as the laser wakefield acceleration (LWFA) mechanism [3] which is a subject of great importance for its potential in developing a new generation of high-energy compact electron accelerators. High-quality electron

\footnotetext{
*Corresponding author: nasr@gist.ac.kr

${ }^{\dagger}$ Corresponding author: leejm@gist.ac.kr
}

beams produced by the LWFA mechanism depend on careful optimization of the laser-plasma parameters such as the laser intensity, pulse duration, focal spot size and quality, plasma density, interaction length, etc. Reasonably large-focal spot, while keeping the intensity above $10^{18}$ $\mathrm{W} / \mathrm{cm}^{2}$, ensures long interaction length, wakefield excitation and electron trapping. Having ultrashort pulses ensures stable propagation of the laser pulse through plasmas, while working at low plasma densities ensures larger acceleration length and, correspondingly, higher maximum electron energies. Generation of $1 \mathrm{GeV}$ monoenergetic electron beam has been observed from the interaction of 40 TW laser pulses with a plasma capillary of $33 \mathrm{~mm}$ length [4]. Supersonic gas jets have been traditionally used as targets for LWFA research because they can support the propagation of the laser beam for long 


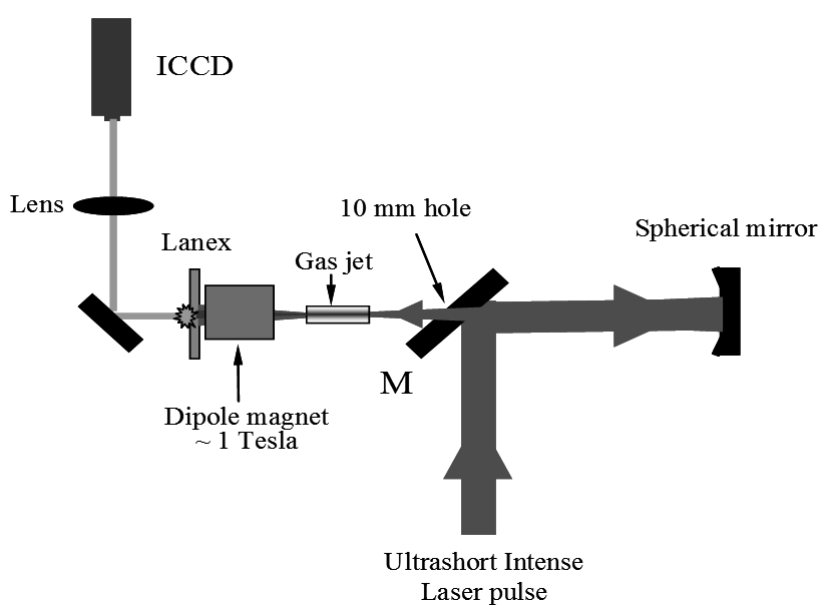

FIG. 1. Schematic diagram of the LWFA experiment at APRI. The laser pulse (27 TW-50 TW) is focused by a spherical mirror onto a supersonic He gas jet. The generated electron beam is diagnosed by using a strong magnet coupled with a lanex screen which is imaged to an ICCD.
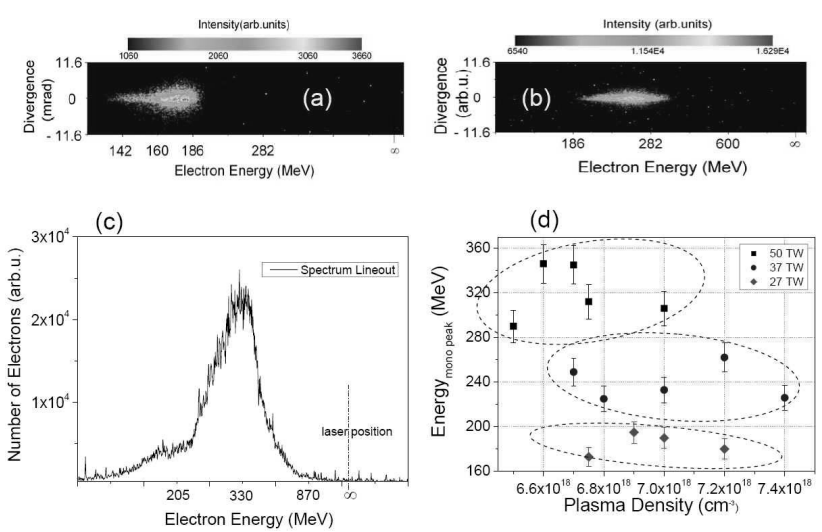

FIG. 2. Quasimonoenergetic electron beams produced in 4-mm long gas jet plasma at various laser powers (27-50 TW). The plasma densities are in the range of $6.5 \times 10^{18}$ $-7.5 \times 10^{18} \mathrm{~cm}^{-3}$. Error bars represent a $\pm 5 \%$ error in the energy measurements and this is applied for all energy values reported in this paper.

enough distances as a result of the effect of self-guiding [5]. They have preference over capillary targets because they are more stable, easily aligned and the simplicity in monitoring the laser-plasma interaction volume. The generation of $100 \mathrm{MeV}$-level quasimonoenergetic electron beams was first observed through the LWFA in gas jet targets [6-8]. Good quality electron beams in the $200 \mathrm{MeV}$ level have been observed in a gas jet target by the laser colliding injection scheme [9].

In this Article, using 100 TW-laser facility we report on the generation of quasimonoenergetic electron beams having energies up to $1 \mathrm{GeV}$ from $\sim 5$-mm long plasma channels. This is the shortest GeV-class electron beam accelerator developed so far. By focusing wave frontcorrected $37 \mathrm{TW}$ laser pulses to a spot size of $22 \mu \mathrm{m}$ through gas jet-plasma having a length of $4 \mathrm{~mm}$ and density of $\sim 7 \times 10^{18} \mathrm{~cm}^{-3}$, stable multi-hundred $\mathrm{MeV}$ quasimonoenergetic electron beams are produced. By increasing the laser power to the $50 \mathrm{TW}$-level, stable electron beams having energies with maximum energies of $1 \mathrm{GeV}$ are observed. Experiments and supporting 3D particle-in-cell (PIC) simulations showed acceleration of multi-electron bunches in a plasma bubble formed in the wake of the laser pulse. Breakthroughs reported in this paper confirm the generation of stable and high quality GeV-class electron beams by a very simple and compact electron accelerator.

\section{EXPERIMENTAL SETUP AND FACILITIES}

\subsection{Laser system}

Our experiments have been carried out at the Center for Femto-Atto Science and Technology (c-FAST) where we have a table-top Ti: sapphire (laser wavelength $\lambda$ $\sim 0.8 \mu \mathrm{m}$ ) laser facility that delivers up to $100 \mathrm{TW}$ laser pulses with a pulse duration of 30 fs and a repetition rate of $10 \mathrm{~Hz}$. The upgrade of this facility to the petawatt level is ongoing. The laser system of APRI and the experimental scheme were presented elsewhere [10]; here we highlight key elements of the setup. A deformable mirror (DM) and wavefront sensor were used to minimize the wavefront aberration of the laser beam (after compression), this led to a significant improvement of the quality of the focal spot and hence the focused intensity [11]. The laser was focused onto the gas jet by a spherical mirror having a focal length of $150 \mathrm{~cm}$, which corresponds to the f-number of 21.4 . The focal spot was nearly Gaussian with a size of $\omega_{0}=22$ $\mu \mathrm{m}(\mathrm{FWHM})$. An ultrafast Pockels cell was installed in the regenerative amplifier in order to reduce the nanosecond pre-pulse level to $\sim 6 \times 10^{-6}$. The laser pulse energy was monitored shot-to-shot and the rms (ratio of the standard deviation to the mean) energy was $9.3 \%$ over 635 shots. A spectral phase interferometry for direct electric field reconstruction (SPIDER) system was used to measure the laser pulse duration daily, and it was $35 \pm 1$ fs.

\subsection{Gas jets}

Two rectangular nozzles having dimensions of $4 \times 1.2$ $\mathrm{mm}^{2}$ and $10 \times 1.2 \mathrm{~mm}^{2}$ were used in our experiments. The two nozzles are Laval-type, and designed via the method of characteristics to produce supersonic flows with Mach number of 5 for the helium gas. Therefore, the gas flow was shock-free, well-localized with sharp boundaries and uniform along the laser propagation direction. Stagnation gas pressures corresponding to plasma densities in the range $3.4 \times 10^{18}$ to $7.4 \times 10^{18} \mathrm{~cm}^{-3}$ are used. The same type of gas jets are widely used in LWFA experiments [14-16]. 


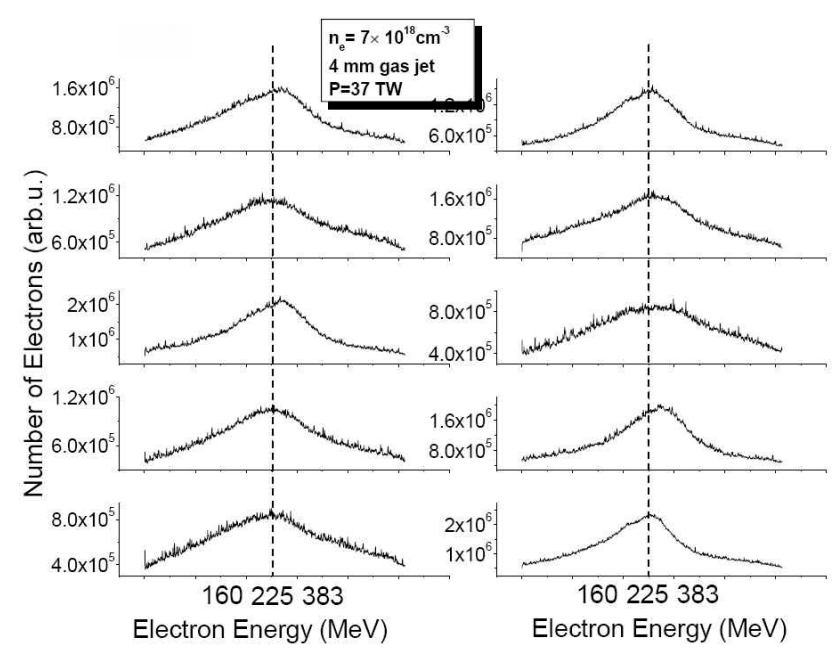

(a)
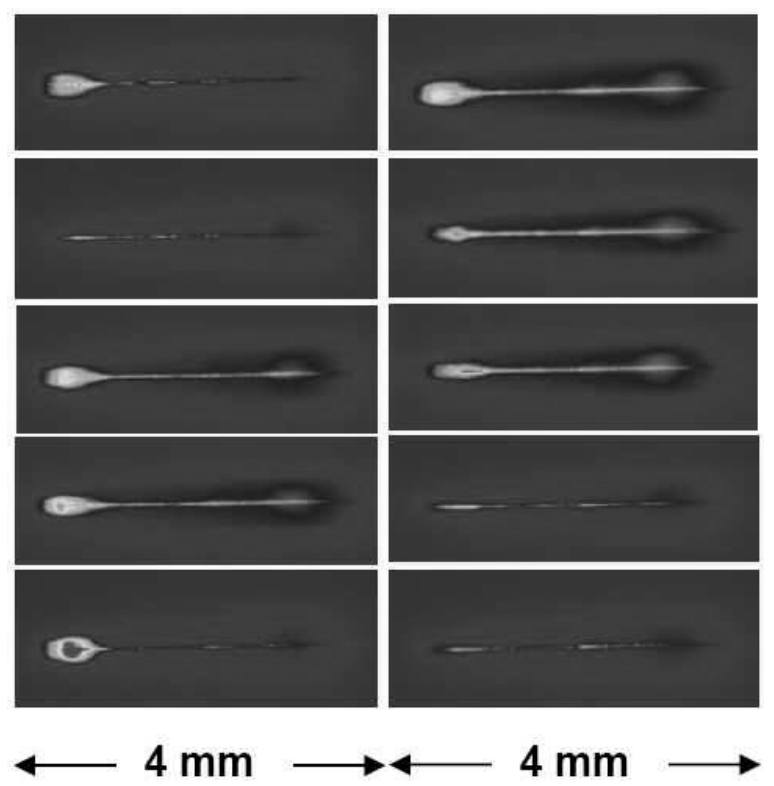

(b)

FIG. 3. (a) Quasimonoenergetic electron beams of ten consecutive shots produced in 4-mm long gas jet at the laser power of $37 \mathrm{TW}$ and plasma density of $7.0 \times 10^{18} \mathrm{~cm}^{-3}$, and (b) the corresponding laser-plasma channles.

\subsection{Electron beam detection}

In the electron detection setup, see Fig. 1, we used a permanent dipole magnet with the following dimensions: $20 \mathrm{~cm}$ long, $7 \mathrm{~cm}$ wide, and $0.9 \mathrm{~cm}$ gap. The field intensity was mapped inside $(0.94 \mathrm{~T})$ and outside of the poles. By taking the edge effect into account, the electron trajectory was calculated by solving the equation of motion numerically. A lanex screen was used for detection of the beam position that corresponds to a certain energy. Detection with an acceptable resolution (of $\sim 5 \%$ ) was achieved for the high-energy electrons in the range of
$140-600 \mathrm{MeV}$. An electron beam of $1 \mathrm{GeV}$ energy makes a total deflection angle of 1.8 degrees at the position of lanex, therefore, electrons with peak energies in the $1 \mathrm{GeV}$-level can be detected but without accurately resolving the energy spread. The total charge of emitted electrons (nC-level) was measured by using an ICT (integrating current transformer) installed midway between the gas jet and the magnet. The high-energy (after magnet) electron beam charge (pC-level) was measured (following the method in Ref. [17]) by counting the number of created photons in the lanex screen (intrinsic conversion efficiency of $16 \% \pm 2$ ) per incident relativistic electron and detected by the ICCD with known gain.

\section{EXPERIMENTAL RESULTS}

\subsection{Scaling of quasimonoenergetic electron beam energies}

In the experiments reported in this paper, three levels of laser power were used, namely $27 \mathrm{TW}, 37 \mathrm{TW}$ and $50 \mathrm{TW}$. The focal spot size was about $22 \mu \mathrm{m}$, obtained with a focusing optic having a focal length of $1.5 \mathrm{~m}$, thus, the laser intensities at the above mentioned powers were $\mathrm{I} \sim 4.7 \times 10^{18}, 6.5 \times 10^{18}$ and $8.8 \times 10^{18} \mathrm{~W} / \mathrm{cm}^{2}$, respectively. At those intensities, the laser normalized vector potentials were $1.6,1.75$, and 2 . The normalized vector potential a0 is defined by $\mathrm{a}_{0}=8.6 \times 10^{-10} \lambda[\mu \mathrm{m}]$ $\mathrm{I}^{1 / 2}\left[\mathrm{~W} / \mathrm{cm}^{2}\right]$. Figure (2) shows raw images obtained by the electron spectrometer of multi-hundred $\mathrm{MeV}$ quasimonoenergetic electron beams generated at various laser and plasma parameters in a 4-mm long gas jet target. At the laser intensity of $4.7 \times 10^{18} \mathrm{~W} / \mathrm{cm}^{2}(27 \mathrm{TW})$ and plasma density of $\mathrm{n}_{\mathrm{e}} \approx 7 \times 10^{18} \mathrm{~cm}^{-3}$ (the plasma wavelength can be estimated as $\lambda_{p}[\mu \mathrm{m}]=3.3 \times 10^{10} /$ $\sqrt{n_{e}\left[\mathrm{~cm}^{-3}\right]} \sim 12.4 \mu \mathrm{m}$ and the dephasing length as $\left.l_{d}=\lambda_{p}^{3} / \lambda^{2} \sim 3 \mathrm{~mm}\right)$, the generated electron beam was quasimonoenergetic with a peak energy of $\sim 180 \mathrm{MeV}$, as shown in Fig. 2 (a). By increasing the laser intensity to $6.5 \times 10^{18} \mathrm{~W} / \mathrm{cm}^{2}(37 \mathrm{TW})$ while the plasma density was in the range of $\approx 6.6 \times 10^{18}-7.4 \times 10^{18} \mathrm{~cm}^{-3}$ we could obtain electron beams having a peak energy of about $260 \mathrm{MeV}$, as shown in Fig. 2(b). Again, by increasing the laser intensity to $8.8 \times 10^{18} \mathrm{~W} / \mathrm{cm}^{2}$ (50 TW) while the plasma density was $\sim 7.0 \times 10^{18} \mathrm{~cm}^{-3}$, the peak energy of the electron beam increased to $306 \mathrm{MeV}$, as shown in the graph Fig. 2(d). The observed energies agree well with previous experimental and simulation results, for approximately similar laser and plasma parameters $[6,18]$. At slightly lower plasma density of $6.6 \times 10^{18} \mathrm{~cm}^{-3}\left(l_{d} \sim 3.3 \mathrm{~mm}\right)$ while the laser intensity was fixed at $8.8 \times 10^{18} \mathrm{~W} / \mathrm{cm}^{2}$, the peak energy of the quasimonoenergetic electron beam reached $350 \mathrm{MeV}$ while its maximum energy reached up to $870 \mathrm{MeV}$, as shown in Fig. 2 (c). The measured total charge of the 


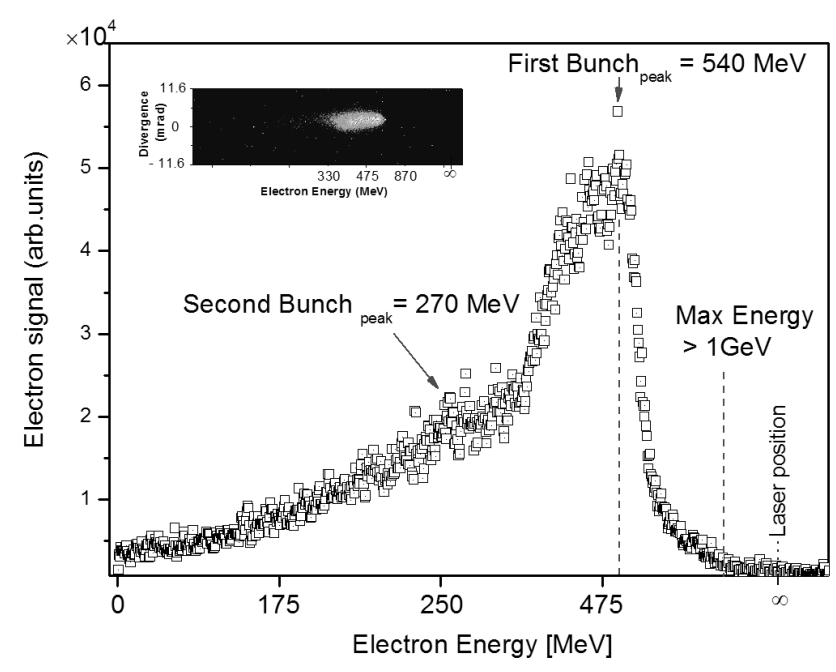

FIG. 4. Quasimonoenergetic electron beam produced in (a) $1 \mathrm{~cm}$-long gas jet plasma at the laser power of 50 TW and plasma density of $3.4 \times 10^{18} \mathrm{~cm}^{-3}$.

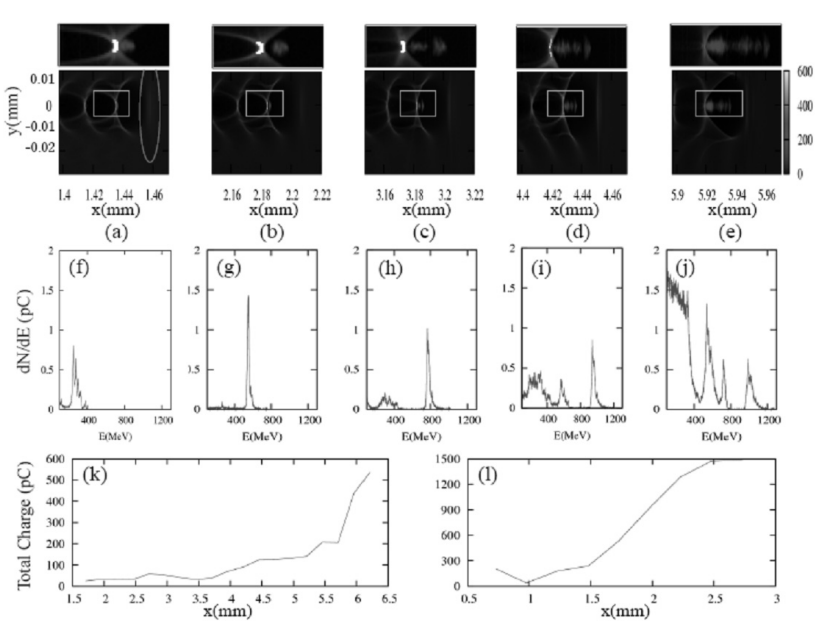

FIG. 5. Three-dimensional PIC simulation results by using the code VORPAL. The plasma electron density distribution and the corresponding energy spectra are shown in (a)-(e) and (f)-(j), respectively. The evolutions of the electron beam charge in the bubble are shown in (k) for the plasma density of $3.4 \times 10^{18} \mathrm{~cm}^{-3}$ and in (l) for the density of $6.8 \times 10^{18} \mathrm{~cm}^{-3}$. The laser pulse propagates from left to right and its position is marked by an ellipse in (a). Areas enclosed in the rectangles are enlarged in the upper row. Figure reprinted with the permission of N. Hafz et al., Nature Photonics 2, 571 (2008). Copyright (2008) Nature Publishing Group.

electron beam in this case was about $500 \mathrm{pC}$ and its divergence angle was about $5 \mathrm{mrad}$ (FWHM), while the most intense part of the electron beam had a divergence of $3.2 \mathrm{mrad}$. The laser-plasma channel lengths were observed to be longer at higher laser intensities due to the self focusing. Channels of lengths $2.5 \mathrm{~mm}, 3.5 \mathrm{~mm}$ and $4 \mathrm{~mm}( \pm 100 \mu \mathrm{m})$ are produced at the laser intensities of $4.7 \times 10^{18} \mathrm{~W} / \mathrm{cm}^{2}, 6.5 \times 10^{18}$ $\mathrm{W} / \mathrm{cm}^{2}$ and $8.8 \times 10^{18} \mathrm{~W} / \mathrm{cm}^{2}$, respectively. Those channel lengths were approximately equal to the dephasing lengths for the plasma densities mentioned above, and this is important for obtaining quasimonoenergetic spectrum $[6,8]$. Experimental data in the graph of Fig. 2 (d) clearly shows the linear scaling of doubling the electron beam energy by doubling the laser power, i.e., the electron beam energy scales as $\sim a_{0}^{2}$ (for given plasma parameters). This is an important experimental result for multi-hundred $\mathrm{MeV}$ electron beam generation by the LWFA scheme.

\subsection{Stability testing of quasimonoenegetic electron beams}

Recent experimental and simulations[5, 10, 19] reported long laser focusing geometries (f/ number 21.4, in our case) are necessary for the stability of laser pulse propagation through the plasma and the generation of stable wakefields and electron beams. Motivated by those predictions, we have performed a stability test for electron beams produced by our laser-plasma accelerator with the following parameters: laser intensity of $6.5 \times 10^{18}$ $\mathrm{W} / \mathrm{cm}^{2}, 4-\mathrm{mm}$ long gas jet and plasma density of $7 \times 10^{18}$ $\mathrm{cm}^{-3}$. Figure 3 (a) shows raw images of the electron beam energy spectra obtained in 10 consecutive shots. The averaged peak energy, and the energy fluctuation were $\sim 237 \mathrm{MeV}$ and $\sim 5 \%$, respectively. The average charge per electron bunch and the charge fluctuation were $\sim 205 \mathrm{pC}$ and $\sim 32 \%$, respectively. We think that the large fluctuation in the electron beam charge was originated from fluctuations in the laser beam direction and spot size which control the electron trapping process [20], however, this topic is currently under investigation. The measured fluctuation in the laser energy during those 10 consecutive shots was $\sim 4.5 \%$ which is very close to the electron beam energy fluctuations. This leads to a conclusion that ultra stable electron beam energies can be realized by stabilizing the shot to shot laser pulse energy. The reproducibility of quasimonoenergetic electron beam generation was $100 \%$. The laserplasma channels, Fig. 3 (b), observed in these 10 shots were almost identical, stable and reproducible; each channel was $\sim 3.5 \mathrm{~mm}$ long. Recent results obtained by the optical injection mechanism [9] through a gas jet showed stable electron beam energy in the $200 \mathrm{MeV}$ range with large charge fluctuation, however this scheme requires colliding two or more ultrashort laser beams (perfectly overlapped in time and space) inside the plasma [21, 22]. Based on single laser pulse LWFA experiments, our stability test shows quite stable quasimonoenergetic electron generation in the energy range of $240 \mathrm{MeV}$.

\subsection{Quasimonoenergetic GeV-class electron beam generation in a low density plasma}


A long gas jet can be very useful for achieving higher electron beam energies if it is operated at significantly low plasma densities $\left(10^{17}-10^{18} \mathrm{~cm}^{-3}\right.$, for example) so that the depahsing length can be of the order of cm-scale. In this case, the full length of a gas jet can be used for electron acceleration if the laser pulse is powerful enough to be guided over the distance. This scenario predicts the generation of a multi-GeV electron beam by using a multi hundred terawatt laser system [19].

In this regime and using tens of terawatt laser pulses, we report on significantly boosting the electron beam energies in a long gas jet operating in the low plasma density regime. In this phase of the experiment, we focused laser pulses having an intensity of $8.8 \times 10^{18}$ $\mathrm{W} / \mathrm{cm}^{2}$ (50 TW) in a 1-cm long supersonic gas jet operating at the plasma density of $\sim 3.4 \times 10^{18} \mathrm{~cm}^{-3}\left(\lambda_{p} \sim 18 \mu \mathrm{m}\right.$ $\left.l_{d} \sim 9 \mathrm{~mm}\right)$. A typical raw image for the electron beam energy spectrum obtained in this case is shown in Fig. 4. The figure shows quasimonoenergetic peak energy of 540 $\mathrm{MeV}$ and maximum energy exceeding $1 \mathrm{GeV}$, significantly larger than the bunch energy obtained in the 4-mm long gas jet at higher plasma density (Fig. $2 \mathrm{c}$ ). In addition to the high-energy bunch shown in Fig 4, there is another lower energy bunch peaked at $270 \mathrm{MeV}$, which is also higher than energy of the second bunch of Fig. 2c. The total beam charge of Fig. 4 is $\sim 200 \mathrm{pC}$, which is lower (but still acceptable) than half the charge of the beam shown in Fig. 2c. This can be understood because a lower plasma density was used for the longer gas jet case.

The observed length of a laser-plasma channel in this case was $\sim 5 \mathrm{~mm}$, which is half the gas jet's length. Thus, the maximum acceleration gradient was approximately 4 orders of magnitude higher than available by conventional RF-based accelerator technologies.

\section{THREE DIMENSIONAL PIC SIMULATIONS AND DISCUSSIONS}

Recently, simulation based -empirical theories have been developed for the laser-plasma bubble regime at very high laser intensities and either low [19] or high plasma densities [23]. However, in our case, the parameters are different, therefore theories of Refs. $[19,23]$ cannot be applied. Thus, we have performed 3D simulations for our problem by using the PIC-code VORPAL [24]. The simulation parameters $(50 \mathrm{TW}$ laser power, $35 \mathrm{fs}$ pulse duration, $24 \mu \mathrm{m}$ spot size, $\mathrm{a}_{0}=2$, plasma densities $3.4 \times 10^{18} \mathrm{~cm}^{-3}$ and $6.8 \times 10^{18} \mathrm{~cm}^{-3}$ and plasma length up to $6.5 \mathrm{~mm}$ ) are chosen to be close to those of the experiment. Figure 5 (except panel l) shows simulation results at the lower plasma density case, $3.4 \times 10^{18} \mathrm{~cm}^{-3}$. First of all, the generation of several plasma bubbles in the wake of the laser pulse was clearly observed in the simulation. Electron injection from the bubble base occurs when the local electron density increases due to the con- tinuous accumulation of electrons. Electron trapping and acceleration took place mostly in the first bubble behind the laser pulse, where the highest wakefield strength exists. Negligible electron trapping was observed in the second or third bubble. We also note that, no clear trapping has been observed in the $2 \mathrm{D}$ simulations at such low-plasma density [18]. The electron injection started at about $1 \mathrm{~mm}$ of propagation distance after some evolution of the laser pulse, which resulted in an increase of laser field amplitude by $\sim 2.3$ times. After a propagation distance of $1.47 \mathrm{~mm}$, the electrons accelerated in the bubble reached a maximum energy of 400 $\mathrm{MeV}$, Figs. 5(a) and 5(f). However, the first electron bunch in the bubble was still not properly formed here, giving a relatively large-energy spread of about $170 \mathrm{MeV}$. As the laser pulse propagates further through the plasma, the peak energy and the monoenergetic features of the first injected bunch were enhanced, Figs. 5(b) and 5(g). The injection of the first electron bunch into the bubble is terminated at the propagation length of $\sim 2 \mathrm{~mm}$ due to the increasing Coulomb force of the trapped electrons, Fig. 5(b). After the injection of the first bunch and its forward acceleration, the injection of another (second) electron bunch started at around $2.4 \mathrm{~mm}$ and it was terminated at $3 \mathrm{~mm}$, Figs. 5(c) and 5(h). Similarly, a third separate electron bunch is injected into the cavity, Figs. 5(d), 5(i). The charge of each subsequent bunch is higher than the charge of its predecessors. Finally, at the distance of $5.2 \mathrm{~mm}$, the injection of the last electron bunch started. This last bunch had large charge (with respect to initial plasma density) to have a $\mathrm{nC}$ level and high trapping rate of electrons, Figs. 5(e) and 5(j). The electron beam's charge evolution in the bubble is shown in Fig. $5(\mathrm{k})$, where the charge increases sharply after $\sim 5 \mathrm{~mm}$ of propagation distance. Due to this feature, the bubble started to elongate, Fig. 5(e) and this resulted in joining of the first and second bubbles to form one large bubble. Then, the first-trapped electron bunch started to experience a deceleration phase of the large bubble's field where no further acceleration is possible. Thus, the effective acceleration range was limited in this case by the bubbles elongation and joining mechanism rather than through the dephasing length of $9 \mathrm{~mm}$ that was estimated through the kinematic approach or by other mechanisms such as laser pulse depletion or diffraction. The rate of acceleration for the secondarytrapped electron bunches in the first cavity was also decreased due to bubble elongation, but still they were accelerated further. After propagating about $5.2 \mathrm{~mm}$, the first quasimonoenergetic electron bunch had a peak energy beyond $1 \mathrm{GeV}$ and a maximum energy of $\sim 1.3$ $\mathrm{GeV}$, a charge of $38 \mathrm{pC}$, and an energy spread of about $2.3 \%$ (half width at half maximum). The second quasimonoenergetic peak had a peak energy of $680 \mathrm{MeV}$ with an energy spread $3.1 \%$ and a charge of $22 \mathrm{pC}$. For the third peak, these parameters are $540 \mathrm{MeV}, 14.5 \%$, and 
$75 \mathrm{pC}$, respectively. Electrons in the last-injected highcharge peak have energies from $35 \mathrm{MeV}$ to $320 \mathrm{MeV}$ at the propagation distance of $5.2 \mathrm{~mm}$.

Simulation results for the higher plasma density of $6.8 \times 10^{18} \mathrm{~cm}^{-3}$ gives almost a similar general scenario for the particle trapping, injection and acceleration. However, due to the high plasma density in this case, the electron trapping started after propagation of $0.5 \mathrm{~mm}$ (earlier than the lower density case). Also, the recovery of the injection occurs much faster than that of the low-density case. The successively trapped electron bunches form almost a continuous electron beam. This is a typical picture for the high-density bubble-acceleration regime $[25,26]$. Figure 5 (l), shows the electron charge evolution in the high-density case. The increase in the trapping rate and the elongation of the cavity started at around $1.5 \mathrm{~mm}$ causing a limitation of the effective acceleration range and maximum energy on the level of $650 \mathrm{MeV}$. In addition, a deterioration of the longitudinal accelerating field was observed due to the beam-loading effect. In future simulations, scanning more interaction parameters will be required in order to achieve a closer understanding to the experimental results, and more attention should be paid to the mechanism of acceleration saturation due to the bubble elongation.

In conclusion, this paper reports significant achievements on laser-driven electron acceleration in the bubble regime. First of all, a doubling of electron beam peak energy in the multi-hundred MeV-range by doubling the laser intensity. Second, a generation of stable 240 $\mathrm{MeV}$-range electron beams. Third, generation of high quality quasimonoenergetic $\mathrm{GeV}$-class electron beams at low plasma density through 5-mm long plasma channel in $1 \mathrm{~cm}$ gas jet. Those results are achieved by using wave-front corrected 27-50 TW, 35 fs laser pulses. Due to their simplicity in alignment and diagnostics, laserplasmas produced on top of supersonic gas jets proved to be the practical targets for compact $100 \mathrm{MeV}-1$ $\mathrm{GeV}$ electron accelerators. Clear features of our results are the stability and achieving the highest acceleration gradients in a laser-driven accelerator. This opens a new way toward the realization of ultra-compact electron accelerators and their applications in science and technology.

\section{ACKNOWLEDGMENT}

We thank S. W. Kang, C. W. Lee and Y.Y. Jin for their technical support. This work was supported by the Ministry of Knowledge and Economy of Korea through the Ultrashort Quantum Beam Facility Program.

\section{REFERENCES}

1. M. D. Perry and G. Mourou, "Terawatt to petawatt subpicosecond lasers," Science 264, 917-924 (1994).

2. G. Mourou, T. Tajima, and S. V. Bulanov, "Optics in the relativistic regime," Rev. Mod. Phys. 78, 309-371 (2006) and references therein.

3. T. Tajima and J. Dawson, "Laser electron accelerator," Phys. Rev. Lett. 43, 267 (1979).

4. W. P. Leemans, B. Nagler, A. J. Gonsalves, Cs. T-1th, K. Nakamura, C. G. R. Geddes, E. Esarey, C. B. Schroeder, and S. M. Hooker, "GeV electron beams from a centimetrescale accelerator,” Nature Physics 2, 696-699 (2006).

5. A. G. R. Thomas, Z. Najmudin, S. P. D. Mangles, C. D. Murphy, A. E. Dangor, C. Kamperidis, K. L. Lancaster, W. B. Mori, P. A. Norreys, W. Rozmus, and K. Krushelnick, "Effect of laser-focusing conditions on propagation and monoenergetic electron production in laserwakefield accelerators," Phys. Rev. Lett. 98, 095004 (2007).

6. J. Faure, Y. Glinec, A. Pukhov, S. Kiselev, S. Gordienko, E. Lefebvre, J.-P. Rousseau, F. Burgy, and V. Malka, "A laser-plasma accelerator producing monoenergetic electron beams," Nature 431, 541-544 (2004).

7. S. P. D. Mangles, C. D. Murphy, Z. Najmudin, A. G. R. Thomas, J. L. Collier, A. E. Dangor, E. J. Divall, P. S. Foster, J. G. Gallacher, C. J. Hooker, D. A. Jaroszynski, A. J. Langley, W. B. Mori, P. A. Norreys, F. S. Tsung, R. Viskup, B. R. Walton, and K. Krushelnick, "Monoenergetic beams of relativistic electrons from intense laser-plasma interactions," Nature 431, 535-538 (2004).

8. G. R. Geddes, Cs. Toth, J. van Tilborg, E. Esarey, C. B. Schroeder, D. Bruhwiler, C. Nieter, J. Cary, and W. P. Leemans, "High-quality electron beams from a laser wakefield accelerator using plasma-channel guiding," Nature 431, 538-541 (2004).

9. J. Faure, C. Rechatin, A. Norlin, A. Lifschitz, Y. Glinec, and V. Malka,"Controlled injection and acceleration of electrons in plasma wakefields by colliding laser pulses," Nature 444, 737-739 (2006).

10. N. Hafz I. W. Choi, J. H. Sung, H. T. Kim, K.-H. Hong, T. M. Jeong, T. J. Yu, V. Kulagin, H. Suk, Y.-C. Noh, D.-K. Ko, and J. Lee, "Dependence of the electron beam parameters on the stability of laser propagation in a laser wakefield accelerator," Appl. Phys. Lett. 90, 151501 (2007).

11. T. M. Jeong, I. W. Choi, N. Hafz, J. H. Sung, S. K. Lee, D.-K. Ko, and J. Lee, "Wavefront correction and customization of focal spot of 100TW Ti:Sapphire laser system," Jap. J. Appl. Phys. 46, 7724-7730 (2007).

12. T. M. Jeong, C. M. Kim, D.-K. Ko, and J. Lee, "Reconstruction of wavefront aberration of 100-TW Ti: sapphire laser pulse using phase retrieval method," J. Opt. Soc. Korea 12, 186-191 (2008).

13. J. H. Lee, Y. C. Lee, and H. J. Cheon, "A circular bimorph deformable mirror for circular/annulus/square laser beam compensation,” J. Opt. Soc. Korea 10, 23-27 (2006).

14. T. Hosokai, K. Kinoshita, A. Zhidkov, K. Nakamura, T. Watanabe, T. Ueda, H. Kotaki, M. Kando, K. Nakajima, and M. Uesaka, "Effect of a laser prepulse on a narrow-cone ejection of $\mathrm{MeV}$ electrons from a gas jet irradiated by an ultrashort laser pulse," Phys. Rev. E 67, 036407 (2003).

15. M. Kando, S. Masuda, A. Zhidkov, A. Yamazaki, H. Kotaki, S. Kondo, T. Homma, S. Kanazawa, K. Nakajima, Y. Hayashi, M. Mori, H. Kiriyama, Y. Akahane, N. Inoue, 
H. Ueda, Y. Nakai, K. Tsuji, Y. Yamamoto, K. Yamakawa, J. Koga, T. Hosokai, M. Uesaka, and T. Tajima, "Electron acceleration by a nonlinear wakefield generated by ultrashort (23-fs) high-peak-power laser pulses in plasma," Phys. Rev. E 71, 015403 (R) (2005).

16. T. Hosokai, K. Kinoshita, A. Zhidkov, A. Maekawa, A. Yamazaki, and M. Uesaka, "Effect of external static magnetic field on the emittance and total charge of electron beams generated by laser-wakefield acceleration," Phys. Rev. Lett. 97, 075004 (2006).

17. Y. Glinec, J. Faure, A. Guemnie-Tafo, V. Malka, H. Monard, J. P. Larbre, V. De Waele, J. L. Marignier, and M. Mostafavi, "Absolute calibration for a broad range single shot electron spectrometer,” Rev. Sci. Inst. 77, 103301 (2006).

18. F. S. Tsung, W. Lu, M. Tzoufras, W. B. Mori, C. Joshi, J. M. Vieira, L. O. Silva, and R. A. Fonseca "Simulation of monoenergetic electron generation via laser wakefield accelerators for 5-25 TW lasers," Phys. Plasmas 13, 056708 (2006).

19. W. Lu, Tzoufras, C. Joshi, F. S. Tsung, W. B. Mori, J. Vieira, R. A. Fonseca, and L. O. Silva "Generating multi-GeV electron bunches using single stage laser wakefield acceleration in a 3D nonlinear regime," Phys. Rev. ST-AB 10, 061301 (2007).
20. M. S. Hur, D. Nandan Gupta, and H. Suk, "Enhanced electron trapping by a static longitudinal magnetic field in laser wakefield acceleration," Phys. Lett. A 372, 2684-2687 (2008).

21. D. Umstadter, J.-K. Kim, and E. Dodd, "Laser injection of ultrashort electron pulses into wakefield plasma waves," Phys. Rev. Lett. 76, 2073 (1996).

22. E. Esarey, R. F. Hubbard, W. P. Leemans, A. Ting, and P. Sprangle "Electron injection into plasma wake fields by colliding laser pulses," Phys. Rev. Lett. 79, 2682-2685 (1997).

23. S. Gordienko and A. Pukhov, "Scaling for ultrarelativistic laser plasmas and quasimonoenergetic electrons," Phys. Plasmas 12, 043109 (2005).

24. C. Nieter and J. R. Cary, "VORPAL : a versatile plasma simulation code,” J. Compu. Phys. 196, 448-474 (2004).

25. A. Pukhov and J. ter. Vehn, "Laser wake field acceleration: the highly non-linear broken-wave regime," Appl. Phys. B. Lasers Opt. 74, 355-361 (2002).

26. N. A. M. Hafz, T. M. Jeong, I. W. Choi, S. K. Lee, K. H. Pae, V. V. Kulagin, J. H. Sung, T. J. Yu, K.-H. Hong, T. Hosokai, J. R. Cary, D.-K. Ko, and J. Lee "Stable generation of $\mathrm{GeV}$-class electron beams from self-guided laser-plasma channels”, Nature Photonics 2, 571-577 (2008). 